\title{
Suspected metastatic adrenocortical carcinoma revealing as pulmonary Kaposi sarcoma in adrenal Cushing's syndrome
}

\author{
Margarita Bala ${ }^{1 *}$, Cristina L Ronchi ${ }^{1}$, Josef Pichl ${ }^{2}$, Vanessa Wild ${ }^{3}$, Stefan Kircher $^{3}$, Bruno Allolio ${ }^{1}$ and Stefanie Hahner ${ }^{1}$
}

\begin{abstract}
Background: Kaposi sarcoma (KS) is a malignant disease most commonly diagnosed in the setting of a human immunodeficiency virus (HIV) infection and in patients receiving immunosuppressive treatment. Pulmonary KS has never been reported in association with endogenous Cushing's syndrome (CS).

Case presentation: A 60-year-old woman presented with symptoms and signs of CS. Adrenal CS was confirmed by standard biochemical evaluation. Imaging revealed a right adrenal lesion (diameter $3.5 \mathrm{~cm}$ ) and multiple pulmonary nodules, suggesting a cortisol-secreting adrenal carcinoma with pulmonary metastases. The patient underwent right adrenalectomy with a pathohistological diagnosis of an adrenal adenoma. Subsequent thoracoscopic wedge resection of one lung lesion revealed pulmonary KS with positive immunostaining for human herpes virus 8 (HHV-8). HIV-serology was negative. Hydrocortisone replacement was initiated for secondary adrenal insufficiency after surgery. Post-operative follow up imaging showed complete remission of all KS-related pulmonary nodules solely after resolution of hypercortisolism.
\end{abstract}

Conclusion: KS may occur in the setting of endogenous CS and may go into remission after cure of hypercortisolism without further specific treatment.

Keywords: Cushing's syndrome, Kaposi sarcoma, Immunosuppression, Hypercortisolism

\section{Background}

Endogenous Cushing's syndrome (CS) is a rare disorder with an incidence of ca. 1/100.000 population [1,2]. The symptoms and signs of CS like truncal obesity, moon face, diabetes mellitus, hypertension and muscle weakness result from chronic glucocorticoid excess. Causes of endogenous hypercortisolism include corticotropin-dependent forms (corticotropic adenoma of the pituitary or ectopic corticotropin secretion) and corticotropin independent forms due to adrenal tumors or primary adrenal hyperplasia [1-3]. The diagnosis is based on the clinical presentation, established biochemical tests (consensus paper) and imaging techniques.

Kaposi sarcoma (KS) is a vascular, highly malignant tumor involving blood and lymphatic vessels [4] with an incidence of ca. 1.2/100.000 (Surveillance Epidemiology

\footnotetext{
* Correspondence: bala_m@ukw.de

${ }^{1}$ Department of Medicine I, Endocrine and Diabetes Unit, University Hospital of Wuerzburg, Oberduerrbacherstrasse 6, Wuerzburg D-97080, Germany Full list of author information is available at the end of the article
}

and End Results Program, Cancer statistics Review 2008-2010). This neoplasm is mostly associated with human herpesvirus 8 (HHV-8) infection, a virus also known as Kaposi-sarcoma-associated Herpes virus (KSHV), which may be transmitted sexually, through blood or saliva, or after organ transplantation [5,6]. A dysregulation of the immune system is frequently involved in the pathogenesis of KS [7]. For this reason, KS became more widely known after the onset of the AIDS epidemic, but it has been also described among patients that underwent organ transplantation and subsequent immunosuppressive treatment $[5,8]$. KS has also been reported after corticosteroid therapy [9]. According to clinical and pathogenetic features, four subtypes of KS are currently distinguished: 1. classic (sporadic or Mediterranean), which mainly affects elderly people, 2. iatrogenic, which is observed in immunosuppressed patients usually after transplantation or after treatment for autoimmune disorders, 3. endemic, in some African countries and 4. epidemic, being human-immunodeficiency-virus (HIV) syndrome-related 
[10]. The therapy varies according to the type of KS. In the case of epidemic (HIV-related) or iatrogenic KS, treatment primarily aims at immune reconstitution by means of highly active antiretroviral therapy (HAART) or reduction of immunosuppressive therapy, respectively $[11,12]$.

We recently experienced the extraordinary case of an adrenal CS associated with pulmonary KS, which reached a complete remission after treatment of CS by adrenalectomy. To our knowledge this is the first reported case of pulmonary KS related to endogenous CS.

\section{Case presentation}

A 60-year-old woman was admitted to our endocrine department with a diagnosis of Cushing's syndrome (CS) presumably caused by a cortisol-secreting adrenal carcinoma with pulmonary metastases. The patient reported a progressive course of central obesity, moon-shaped face, muscle atrophy and weakness, asthenia and emotional disturbance over the last six years. During this period she had developed arterial hypertension, which was poorly controlled despite five antihypertensive drugs. Five years ago abdominal magnetic resonance (MR) imaging had been performed in the local hospital for further evaluation of a suspected liver hemangioma, and revealed an incidental finding of a $2.5 \mathrm{~cm}$ lesion in the right adrenal. No endocrinological evaluation was performed. Three years before admission she underwent abdominal computed tomography $(\mathrm{CT})$ imaging for evaluation of recurrent hypertensive crises. No change of the adrenal tumor size was found. Screening for pheochromocytoma and primary hyperaldosteronism was negative. In the weeks before presentation at our department the patient complained of polyuria and exertional dyspnoe. The family physician diagnosed diabetes mellitus, which was treated with dietary measures. Furthermore, abdominal CT imaging was repeated, which showed an increase of the adrenal tumor size to $3.3 \mathrm{~cm}$. The patient was subsequently admitted to the local hospital for further endocrinological investigation.
Laboratory tests performed at the local hospital showed failure to suppress cortisol levels after $1 \mathrm{mg}$ dexamethasone and elevated 24-hour urine cortisol secretion. Plasma adrenocorticotropin (ACTH) levels were suppressed indicating an adrenal origin of the hypercortisolism (Table 1). A pulmonary nodule was suspected in the abdominal CT imaging and an additional chest CT revealed multiple bilateral pulmonary nodules which were not described in a CT imaging of the chest performed 5 years earlier. A fluorodeoxyglucose positron emission tomography (FDG-PET) was performed for further evaluation. A high uptake of ${ }^{18}$ F-FDG for both the adrenal lesion and the pulmonary nodules was observed (Figure 1 and Figure 2).

The patient presented in our clinic for further evaluation of the suspected metastatic adrenal carcinoma. The physical examination revealed skin atrophy with bruises, buffalo hump, central obesity and facial hirsutism. Laboratory tests confirmed the presence of ACTHindependent hypercortisolism (Table 1). For histological verification and for "debulking" purposes to potentially reduce the cortisol excess, the patient underwent a right laparoscopic adrenalectomy.

Unexpectedly, the histological report indicated an adrenocortical adenoma with no evidence of malignancy. The tumor size was $3.5 \mathrm{~cm}$ and the Weiss score was 0 , compatible with a definitive diagnosis of a benign cortisol-secreting adrenocortical tumor (Figure 3). Postoperatively signs of CS gradually receded and laboratory testing revealed secondary adrenal insufficiency (Table 1). The patient received intra- and postoperative glucocorticoid coverage followed by chronic replacement therapy for adrenal insufficiency. A thoracoscopic wedge resection with biopsies of the left upper and lower lobe was performed. Histopathology showed spindle-shaped cells with positive immunostaining for CD31, CD34 and for human herpes virus 8 (HHV-8) (Figure 4), consistent with a diagnosis of pulmonary Kaposi's sarcoma. Neither skin lesions nor involvement of the gastrointestinal

Table 1 Laboratory and endocrine baseline and function tests of the patient before and after adrenalectomy

\begin{tabular}{|c|c|c|c|}
\hline Laboratory tests & Preoperative values & Postoperative values & Normal range \\
\hline Lymphocyte count $\left({ }^{*} 1000 / \mu l\right)$ & 1.26 & 2.89 & $1-4.05$ \\
\hline Lymphocyte (\%) & 12.9 & 30.6 & $25-40$ \\
\hline Cortisol basal $(\mu \mathrm{g} / \mathrm{dl})$ & 27 & 3.9 & $5-25$ \\
\hline Cortisol after 1 mg Dexamethason-Suppression-Test $(\mu \mathrm{g} / \mathrm{dl})$ & 18 & 3.5 & $<1.8$ \\
\hline Cortisol after short synacthen-Stimulation-Test $(\mu \mathrm{g} / \mathrm{dl})$ & Not applicable & 10.8 & $>18$ \\
\hline 24-hour urine cortisol ( $\mu \mathrm{g} / \mathrm{L})$ & 270 & Not applicable & $8-70$ \\
\hline Midnight salivary cortisol $(\mu \mathrm{g} / \mathrm{dl})$ & 1.35 & 0.09 & $0-0.15$ \\
\hline ACTH (pg/ml) & 8.9 & 6.9 & $0-46$ \\
\hline
\end{tabular}

$\mathrm{ACTH}=$ adrenocorticotropic hormone. Bold $=$ altered parameters.

${ }^{*}=$ multiplied by. 


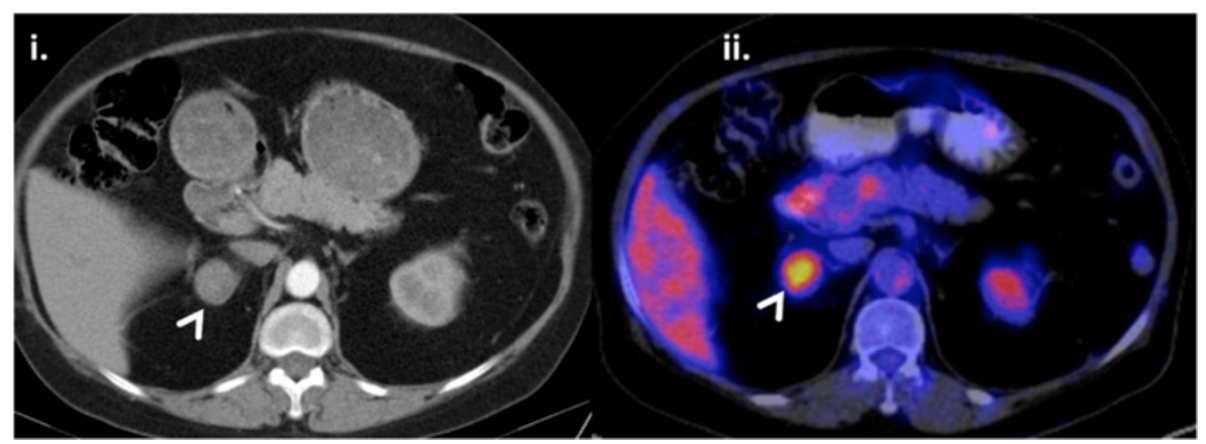

Figure 1 Imaging of the right adrenal tumor at initial evaluation. i. CT imaging revealed a $3.3 \mathrm{~cm}$ right adrenal tumor (white arrow) ii. High uptake of ${ }^{18} \mathrm{~F}$-FDG with standardized uptake value (SUV) of 5.0 for the right adrenal lesion (white arrow).

tract or other organs were detected. Gastroscopy and colonoscopy were unremarkable. Human immunodeficiency virus (HIV) testing was negative and testing of the immune status showed normal CD4 cell counts (1014 cells $/ \mathrm{mm}^{3}$; normal range: $410-1590$ cells $/ \mathrm{mm}^{3}$ ) as well as a normal CD4/CD8 ratio (1.18; normal range: 0.8-4.2) eight weeks after adrenalectomy. The differential white blood cell count revealed relative lymphopenia before the adrenalectomy which was normalised 8 weeks after adrenalectomy (Table 1).

Palliative treatment with systemic combination chemotherapy was considered after the diagnosis of advanced KS was established, but was refused by the patient. The patient experienced significant clinical improvement of her hypercortisolism-related clinical symptoms such as normalization of hypertension under antihypertensive medication, fat redistribution and gradual recovery of muscular strength. Restaging was performed with chest and abdominal CT three months (Figure 5) and with FDG-PET/CT six months after the adrenalectomy and showed complete disappearance of all KS-induced pulmonary nodules.

\section{Discussion}

We here describe a case of adrenal CS associated with pulmonary KS with complete remission after successful treatment of CS by unilateral adrenalectomy. It is well known that cortisol excess causes a state of immunosuppression $[13,14]$. KS occurs in patients with an immunocompromised state mainly due to advanced HIV disease or in transplant patients $[4,8]$. The etiologic agent of almost all forms of KS is HHV-8, but additional immunosuppression is necessary for the manifestation of this malignant disease [5]. Corticosteroids seem to play a role in the development of KS. Classical KS risk was found to be strongly and independently associated with oral corticosteroid use [15]. In addition, KS has also been reported in association with the immunosuppressive effects of systemic steroids in various clinical conditions (iatrogenic KS). After withdrawal or reduction of corticosteroids a partial or complete regression of KS may occur [16-19]. The exact pathophysiological mechanism of this association is not yet understood. The increased incidence and severity of KS in transplant recipients was not found to be associated with direct cellular effects of corticosteroids on HHV8 reactivation [20]. Glucocorticoids seem however to induce KS cell proliferation through the regulation of transforming growth factor- $\beta$ [21]. Furthermore, in AIDSKS tumor biopsies high expression of glucocorticoid receptors (GRs) was detected as well as an up-regulation of GRs by KS-growth-promoting factors like tumor necrosis factor- $\alpha$ [22].

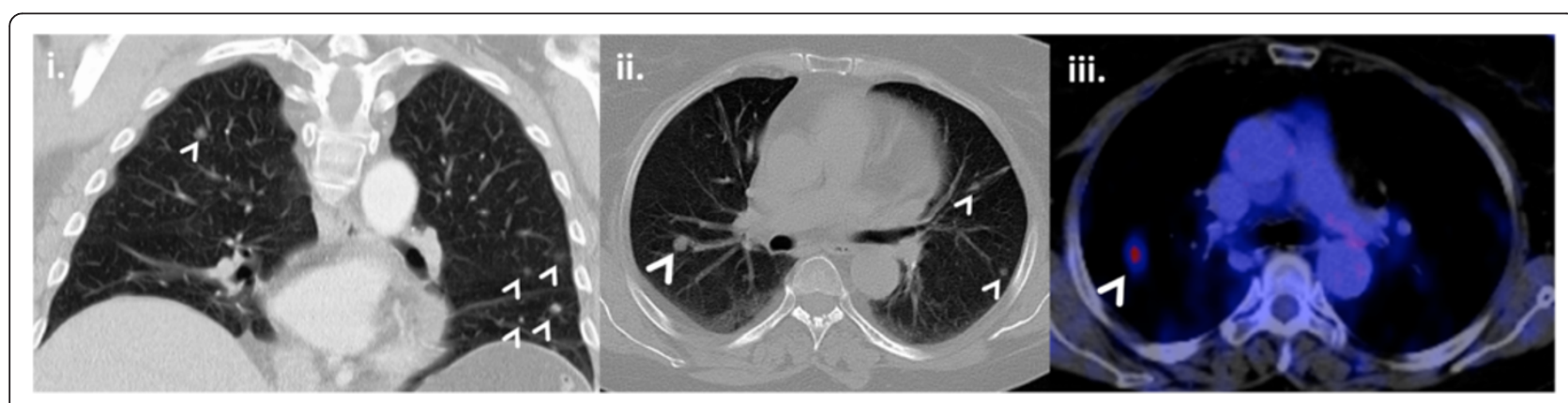

Figure 2 Imaging of the bilateral pulmonary nodules at initial evaluation. i. and ii. Multiple bilateral pulmonary nodules (white arrows) in CT imaging iii. High uptake of ${ }^{18}$ F-FDG in one pulmonary nodule (white arrow) (SUV of 3.7), highly suspicious for malignancy. 


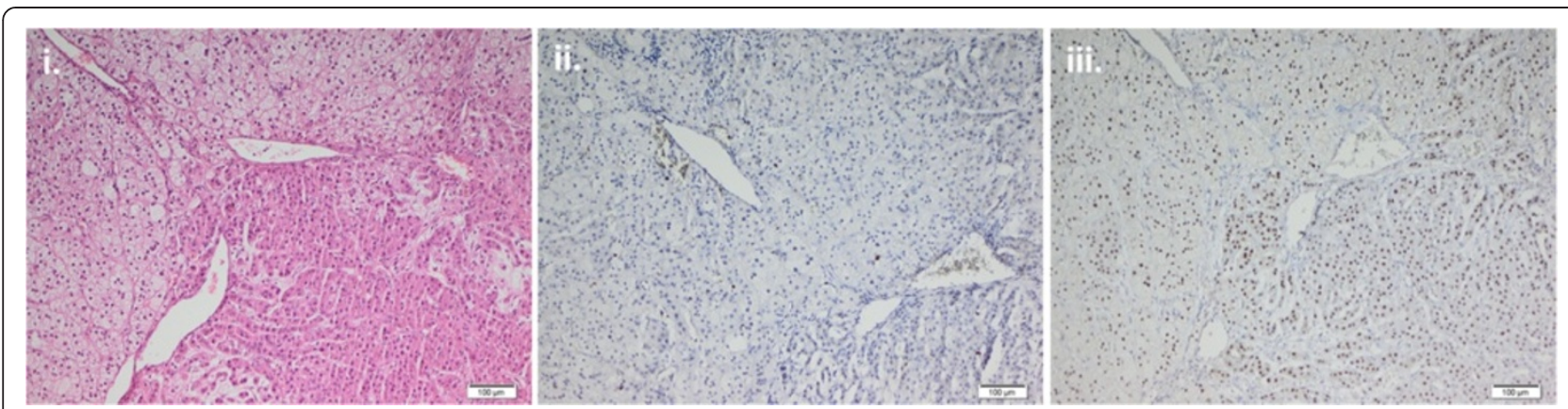

Figure 3 Paraffin sections with immunohistochemical staining of the cortisol-secreting adrenal lesion. i. H-E staining ii. Negative staining of Ki67 iii. Positive nuclear staining of SF1.

A literature search revealed two cases of endogenous ACTH dependent CS which both were associated with cutaneous KS [23,24]. In the first case a 43-year-old HIV-negative woman from Turkey developed CS due an ectopic hypothalamic adrenocorticotropic hormonesecreting adenoma. The patient developed diffuse skin lesions on her abdomen, legs, eyelids, and toes, which were proven to be KS by a skin biopsy. Within one month after transcranial surgery and cure of CS, all skin lesions disappeared spontaneously [23]. In the second case a 54-year-old Hispanic HIV-negative man developed CS due to a pituitary ACTH-secreting adenoma. Coincidentally he showed numerous raised, purplish, non-blanching plaques extended throughout his lower extremities, which were also proven to be KS by skin biopsy. He underwent repeated transphenoidal surgeries followed by bilateral adrenalectomy. The patient received additional treatment with systemic combination chemotherapy with liposomal doxorubicin and paclitaxel resulting in a significant decrease of the KS lesions [24].

In our case the patient had adrenal CS and presented at a late stage of KS (pulmonary Kaposi) indicating longer term severe immunosuppression. Clinical symptoms of CS were present for at least 5 years and an incidental adrenal lesion had already been diagnosed in MR imaging 5 years before. CS is associated with immune dysregulation and CS patients have been shown to display impairment in immune regulation, including changes in both the percentage of specific lymphocyte subsets and specific lymphocyte functional activities [25]. The patient presented in our clinic for further evaluation of suspected metastatic adrenal carcinoma, therefore we did not initially perform any specific preoperative examination of the patient's immune system. After establishment of the diagnosis of Kaposi Sarcoma, specific testing of the immune status showed normal CD4 cell counts and normal CD4/CD8 ratio. However, testing had been performed eight weeks after adrenalectomy and after resolution of the hypercortisolism. Treatment of CS resulted in a complete remission of KS despite a visceral manifestation which is usually associated with a poor prognosis [26]. This demonstrates a key role of cortisol excess in the pathogenesis and the high relevance of restoration of immune function for treatment of KS.

Our case also emphasizes the importance of hormonal workup in adrenal incidentalomas including screening
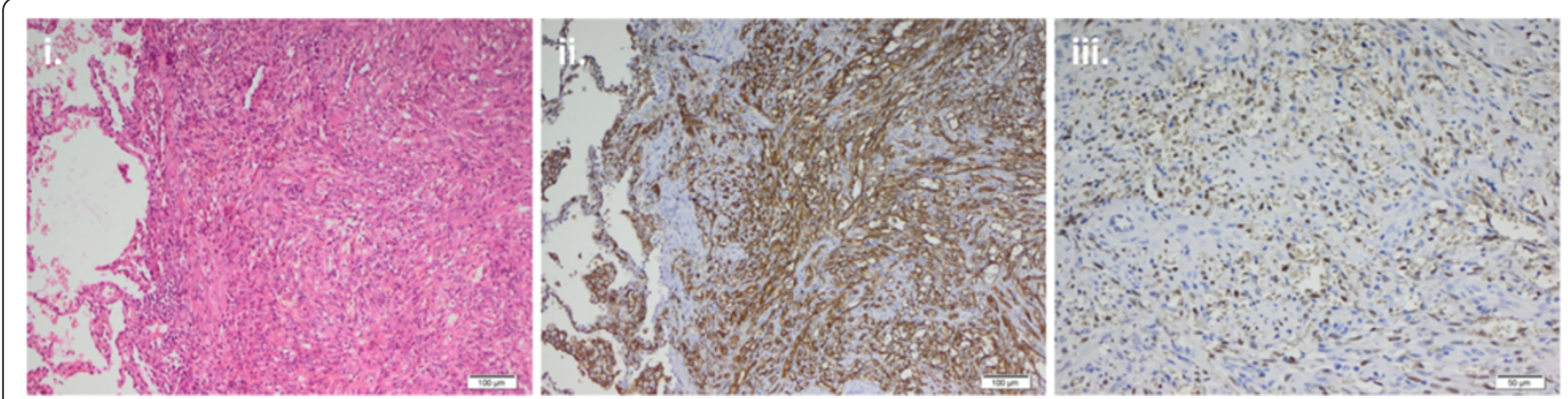

Figure 4 Paraffin sections with immunohistochemical staining of the left lobe lung biopsy. i. H-E staining ii. Positive membranous and cytoplasmic staining of CD31 iii. Positive nuclear staining of human herpes virus 8 (HHV-8). 


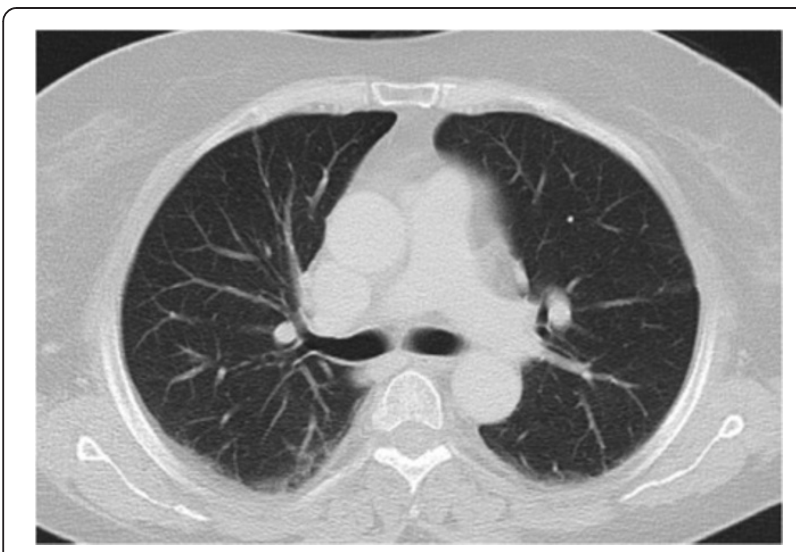

Figure 5 Restaging with CT three months after the adrenalectomy showed disappearance of all KS-induced pulmonary nodules. for cortisol-producing adenoma, hyperaldosteronism and pheochromocytoma which was delayed and only incompletely performed in our patient.

The combination of cortisol excess with positive FDGuptake of both the adrenal tumor and the pulmonary nodules clearly suggested a cortisol-secreting adrenal carcinoma with pulmonary metastases. Malignant adrenal lesions have a higher standardized uptake value (SUV) than benign lesions, but in most cases have a tumor diameter $>5 \mathrm{~cm}$ [27]. Moreover, a high SUV is occasionally also found in benign hormone secreting adenomas [27]. Furthermore, the long standing history of an adrenal incidentaloma in our patient also pointed to the possibility of a benign tumor, although some increase in diameter was documented prior admission. These aspects supported the indication for a removal of the adrenal tumor which otherwise would have remained a matter of debate in stage IV ACC [28].

Currently, four different types of $\mathrm{KS}$ are distinguished, the classic, endemic, epidemic HIV related and the iatrogenic type which is related to treatment with immunosuppressants. Iatrogenic KS shows similar features as have been observed in the patients with endogenous CS. Based on our case and the two earlier reports of cutaneous $\mathrm{KS}$ in ACTH dependent CS, we suggest adding a fifth type of KS with endogenous corticosteroid-excess as cause of KS (Table 2) being, however, closely related to the iatrogenic phenotype. The association of KS with both exogenous and endogenous glucocorticoid excess requires a concurrent HHV-8 infection. Similar to KS in association with iatrogenic immunosuppression, which may resolve completely after reduction or discontinuation of immunotherapy, effective treatment of endogenous hypercortisolism may also successfully lead to remission of KS.

\section{Conclusions}

Our findings demonstrate that endogenous immunosuppression due to Cushing's syndrome may lead to pulmonary Kaposi sarcoma which may regress after treatment of hypercortisolism. The data emphasize the key role of glucocorticoid excess in the pathogenesis of Kaposi Sarcoma and the high relevance of restoration of immune function for its treatment. The data further emphasize the need of careful evaluation of patients with adrenal lesions.

\section{Consent}

Written informed consent was obtained from the patient for publication of this Case report and any accompanying images. A copy of the written consent is available for review by the Editor of this journal.

Table 2 Clinical types of Kaposi sarcoma (KS)

\begin{tabular}{|c|c|c|c|}
\hline Type of KS & $\begin{array}{l}\text { Cutaneous } \\
\text { manifestation }\end{array}$ & $\begin{array}{l}\text { Visceral } \\
\text { manifestation }\end{array}$ & Clinical course \\
\hline $\begin{array}{l}\text { Classic } \\
\text { (sporadic or Mediterranean) }\end{array}$ & $\begin{array}{l}\text { Yes } \\
\text { (often limited to } \\
\text { lower extremity) }\end{array}$ & $\begin{array}{l}\text { No } \\
\text { (uncommon) }\end{array}$ & Low malignant potential \\
\hline $\begin{array}{l}\text { Endemic } \\
\text { (African) }\end{array}$ & Yes & Yes & Indolent, occasionally aggressive progression \\
\hline Epidemic AIDS-associated & Yes & Yes & $\begin{array}{l}\text { Indolent, occasionally aggressive progression. } \\
\text { May regress with HIV treatment }\end{array}$ \\
\hline $\begin{array}{l}\text { Latrogenic } \\
\text { (immunosuppression-related) }\end{array}$ & Yes & Yes & $\begin{array}{l}\text { May heal spontaneously with reduction or } \\
\text { discontinuation of immunosuppression. } \\
\text { May be aggressive. }\end{array}$ \\
\hline $\begin{array}{l}\text { Endogenic } \\
\text { (endogenous corticosteroid-excess-related) }\end{array}$ & $\begin{array}{l}\text { Yes } \\
\text { (in the literature) }\end{array}$ & $\begin{array}{l}\text { Yes } \\
\text { (in our case) }\end{array}$ & May regress after treatment of hypercortisolism \\
\hline
\end{tabular}




\section{Competing interests}

The authors declare that they have no competing interests.

\section{Authors' contributions}

MB, CLR and SH treated the patient and drafted the article. JP initially treated the patient. W and SK carried out the immunoassays. BA critically revised the manuscript. All authors read and approved the final manuscript.

\section{Acknowledgments}

We would like to thank Prof. M. Cordes (Center of radiology and nuclear medicine, Nuremberg) for providing us with the fluorodeoxyglucose positron emission tomography (FDG-PET) imaging.

Stefanie Hahner is an awardee of the Else Kröner-Fresenius Stiftung (2010EKES.29). This publication was funded by the German Research Foundation (DFG) and the University of Wuerzburg in the funding programme Open Access Publishing.

\section{Author details}

'Department of Medicine I, Endocrine and Diabetes Unit, University Hospital of Wuerzburg, Oberduerrbacherstrasse 6, Wuerzburg D-97080, Germany. ${ }^{2}$ Department of Internal Medicine, St. Theresien Hospital of Nuremberg, Nürnberg, Germany. ${ }^{3}$ Department of Pathology, University of Wuerzburg, Würzburg, Germany.

Received: 18 March 2014 Accepted: 23 July 2014

Published: 30 July 2014

\section{References}

1. Lindholm J, Juul S, Jorgensen JO, Astrup J, Bjerre P, Feldt-Rasmussen U, Hagen C, Joergensen J, Kosteljanetz M, Kristensen L, Laurberg P, Schmidt K, Weeke J: Incidence and late prognosis of Cushing's syndrome: a population-based study. J Clin Endocrinol Metab 2001, 86(1):117-123.

2. Etxabe J, Vazquez JA: Morbidity and mortality in Cushing's disease: an epidemiological approach. Clin Endocrinol (Oxf) 1994, 40(4):479-484.

3. Boscaro M, Barzon L, Fallo F, Sonino N: Cushing's syndrome. Lancet 2001, 357(9258):783-791.

4. Martin RW 3rd, Hood AF, Farmer ER: Kaposi sarcoma. Medicine 1993, 72:245-261.

5. Gao SJ, Kingsley L, Hoover DR, Spira TJ, Rinaldo CR, Saah A, Phair J, Detels R, Parry P, Chang Y, Moore PS: Seroconversion to antibodies against Kaposi's sarcoma-associated herpesvirus-related latent nuclear antigens before the development of Kaposi's sarcoma. N Engl J Med 1996, 335(4):233.

6. Schulz TF: KSHV (HHV8) infection. J Infect 2000, 41(2):125-129.

7. Crispo A, Tamburini M, De Marco MR, Ascierto P, Silvestro P, Ronga D, Tridente V, Desicato S, Carbone S, Fabbrocini G, Spiteri D, Montella M: HHV-8 prevalence, immunosuppression and Kaposi's sarcoma in South Italy. Int J Mol Med 2001, 7(5):535-538.

8. Shepherd FA, Maher E, Cardella C, Cole E, Greig P, Wade JA, Levy G: Treatment of Kaposi's sarcoma after solid organ transplantation. J Clin Oncol 1997, 15(6):2371-2377.

9. Trattner A, Hodak E, David M, Sandbank M: The appearance of Kaposi sarcoma during corticosteroid therapy. Cancer 1993, 72(5):1779-1783.

10. Tappero JW, Conant MA, Wolfe SF, Berger TG: Kaposi's sarcoma: epidemiology, pathogenesis, histology, clinical spectrum, staging criteria and therapy. J Am Acad Dermatol 1993, 28:371-395.

11. Mosam A, Shaik F, Uldrick TS, Esterhuizen T, Friedland GH, Scadden DT, Aboobaker J, Coovadia HM: A randomized controlled trial of highly active antiretroviral therapy versus highly active antiretroviral therapy and chemotherapy in therapy-naive patients with HIV-associated Kaposi sarcoma in South Africa. J Acquir Immune Defic Syndr 2012, 60(2):150-157.

12. Hosseini-Moghaddam SM, Soleimanirahbar A, Mazzulli T, Rotstein C, Husain S: Post renal transplantation Kaposi's sarcoma: a review of its epidemiology, pathogenesis, diagnosis, clinical aspects, and therapy. Transpl Infect Dis 2012, 14(4):338-345.

13. Graham BS, Tucker WS Jr: Opportunistic infections in endogenous Cushing's syndrome. Ann Intern Med 1984, 101(3):334-338.

14. Bakker RC, Gallas PR, Romijn JA, Wiersinga WM: Cushing's syndrome complicated by multiple opportunistic infections. J Endocrinol Invest 1998, 21(5):329-333.

15. Anderson LA, Lauria C, Romano N, Brown EE, Whitby D, Graubard BI, Li Y, Messina A, Gafà L, Vitale F, Goedert JJ: Risk factors for classical Kaposi sarcoma in a population-based case-control study in Sicily. Cancer Epidemiol Biomarkers Prev 2008, 17(12):3435-3443.

16. Nassar D, Schartz NE, Bouché C, Levy A, Kerob D, Agbalika F, Lafaurie M, Lebbe C: Kaposi's sarcoma after long-acting steroids: time until remission and drug washout. Dermatology 2010, 220:159-163.

17. Antón E: Kaposi's sarcoma in an immunocompetent patient following corticosteroid therapy. Eur J Intern Med 2008, 19:226.

18. Joo M, Soon Lee S, Jin Park H, Shin HS: latrogenic Kaposi's sarcoma following steroid therapy for nonspecific interstitial pneumonia with HHV-8 genotyping. Pathol Res Pract 2006, 202(2):113-117.

19. Kondo Y, Izumi T, Yanagawa T, Kanda H, Katano H, Sata T: Spontaneously regressed Kaposi's sarcoma and human herpesvirus 8 infection in a human immunodeficiency virus-negative patient. Pathol Int 2000, 50(4):340-346.

20. Zoeteweij JP, Rinderknecht AS, Davis DA, Yarchoan R, Blauvelt A: Minimal reactivation of Kaposi's sarcoma-associated herpesvirus by corticosteroids in latently infected B cell lines. J Med Virol 2002, 66(3):378-383.

21. Cai J, Zheng T, Lotz M, Zhang Y, Masood R, Gill P: Glucocorticoids induce Kaposi's sarcoma cell proliferation through the regulation of transforming growth factor- $\beta$. Blood 1997, 89:1491-1500.

22. Guo WX, Antakly T, Cadotte M, Kachra Z, Kunkel L, Masood R, Gill P: Expression and cytokine regulation of glucocorticoid receptors in Kaposi's sarcoma. Am J Pathol 1996, 148(6):1999-2008.

23. Yetkin DO, Kafadar A, Gazioglu N, Oz B, Kaner G, Oguz O, Kadioglu P: Kaposi sarcoma related to an ectopic hypothalamic adrenocorticotropic hormone-secreting adenoma: case report. Neurosurgery 2009, 65(6 Suppl):E110-E111. discussion E111.

24. Jeng L, Rios A, Ruppe MD: Kaposi sarcoma in the setting of cushing disease. Endocr Pract 2011, 17(4):e87-e91.

25. Kronfol Z, Starkman M, Schteingart DE, Singh V, Zhang Q, Hill E: Immune regulation in Cushing's syndrome: relationship to hypothalamic-pituitaryadrenal axis hormones. Psychoneuroendocrinology 1996, 21(7):599-608.

26. Vogt T, Brockmeyer N, Kutzner H, Schöfer H: Brief S1 guidelines-Cutaneous angiosarcoma and Kaposi sarcoma. J Dtsch Dermatol Ges 2013, 11(Suppl 3):2-9. 2-10.

27. Ansquer C, Scigliano S, Mirallié E, Taïeb D, Brunaud L, Sebag F, Leux C, Drui D, Dupas B, Renaudin K, Kraeber-Bodéré F: 18 F-FDG PET/CT in the characterization and surgical decision concerning adrenal masses: a prospective multicentre evaluation. Eur J Nucl Med Mol Imaging 2010, 37(9):1669-1678.

28. Berruti A, Baudin E, Gelderblom H, Haak HR, Porpiglia F, Fassnacht M, Pentheroudakis G: ESMO Guidelines Working Group. Adrenal cancer: ESMO Clinical Practice Guidelines for diagnosis, treatment and follow-up. Ann Oncol 2012, 23(Suppl 7):vii131-vii138.

\section{doi:10.1186/1472-6823-14-63}

Cite this article as: Bala et al: Suspected metastatic adrenocortical carcinoma revealing as pulmonary Kaposi sarcoma in adrenal Cushing's syndrome. BMC Endocrine Disorders 2014 14:63.

\section{Submit your next manuscript to BioMed Central and take full advantage of:}

- Convenient online submission

- Thorough peer review

- No space constraints or color figure charges

- Immediate publication on acceptance

- Inclusion in PubMed, CAS, Scopus and Google Scholar

- Research which is freely available for redistribution 